\title{
HIV-1 and HCV sequences from Libyan outbreak
}

In 1998, outbreaks of human immunodeficiency virus type 1 (HIV-1) and hepatitis C virus (HCV) infection were reported in children attending Al-Fateh Hospital in Benghazi, Libya. Here we use molecular phylogenetic techniques to analyse new virus sequences from these outbreaks. We find that the HIV-1 and HCV strains were already circulating and prevalent in this hospital and its environs before the arrival in March 1998 of the foreign medical staff (five Bulgarian nurses and a Palestinian doctor) who stand accused of transmitting the HIV strain to the children.

Almost half of the 111 children studied in the early months after the discovery of the outbreak showed evidence of both HIV-1 and HCV infection ${ }^{1}$. Of 418 children eventually affected by these viruses, 248 were referred to European hospitals ${ }^{12}$. Sequence analysis of 51 children dassified the HIV-1 infection as the strain CRF02_AG; HCV infection was classified as genotype 4 or subtype $1 \mathrm{a}$ in 15 children $^{1,2}$.

We studied HIV-1 gag gene sequences from 44 affected children, plus $61 \mathrm{HCV}$ E1E2 gene sequences that span the HCV hypervariable region (for methods, see supplementaryinformation). By using these data in an evolutionary analysis, we could place a real timescale on the transmission history of the outbreaks.

We collated all available reference strains that were closely related to the sequences from the Al-Fateh Hospital, then estimated and assessed phylogenies using algorithmic, bayesian and maximum-likelihood methods (for details, see supplementary information). The HIV-1 sequences from the hospital form a well supported monophyletic cluster within the CRF02_AG clade, indicating that the outbreak arose from one CRF02_AGlineage. The cluster is closest to three west African reference sequences (Fig. 1a), the basal location of which suggests that the Al-Fateh Hospital lineage arrived in Libya from there. The branch length leading to the Al-Fateh Hospital cluster is perfectly typical; hence the Al-Fateh Hospital strain is not unusually divergent ${ }^{2}$.

In an equivalent $\mathrm{HCV}$ phylogenetic analysis, the HCV sequences from the hospital formed three monophyletic clusters containing 11 subtype-4a sequences, phylogenetically placed among Egyptian subtype 4a lineages; 22 sequences most closely related to a Cameroonian genotype- 4 strain; and 24 sequences belonging to the worldwide and prevalent subtype 1a; four remaining sequences belong to genotype 4 (Fig. 1b, c, see supplementary information).

Epidemiological linkage of the HIV-1 and $\mathrm{HCV}$ clusters from Al-Fateh Hospital with sequences from sub-Saharan Africa is to be expected, given the large number of migrants within or passing through Libya ${ }^{3}$; indeed, the Libyan authorities have expressed concern about the risk of introduction of HIV/AIDS and hepatitis as a result of this migration 4 . In addition, $\mathrm{HCV}$ genotype 4 is endemic to central Africa and the Middle East ${ }^{5-7}$, and subtype $4 \mathrm{a}$ is exceptionally prevalent in neighbouring Egypt ${ }^{8,9}$.
Virus sequences also contain temporal information about the date of origin and age of epidemics ${ }^{10}$. We therefore comprehensively analysed the evolution of the Al-Fateh Hospital clusters using an established bayesian Markow chain Monte Carlo (MCMC) approach ${ }^{9,10}$ that appropriately accounts for estimation uncertainty. We estimated three parameter values for each cluster: the date of its most recent common ancestor; the probability that its most recent common ancestor was more recent than 1 March 1998; and the percentage of its lineages that already existed before 1 March 1998. (These values are conservative, because cluster origins could be older than the most recent common ancestor, but not younger.) To avoid model selection bias, we used a range of applicable models.

We found that, irrespective of which model was used, the estimated date of the most common recent ancestor for each cluster predated March 1998, sometimes by many years (Fig. 2).

In most analyses, the probability that the clusters from the Al-Fateh Hospital originated after that time was almost zero (for details, see supplementary information). For the three HCV clusters, the percentage of lineages already present before March 1998 was about $70 \%$; the equivalent percentage for the HIV-1 cluster was estimated at about $40 \%$.

Our results support the existing nosocomial transmission scenario ${ }^{1,11}$ and suggest that AlFateh Hospital had a long-standing infection-control problem. The earlier origin and

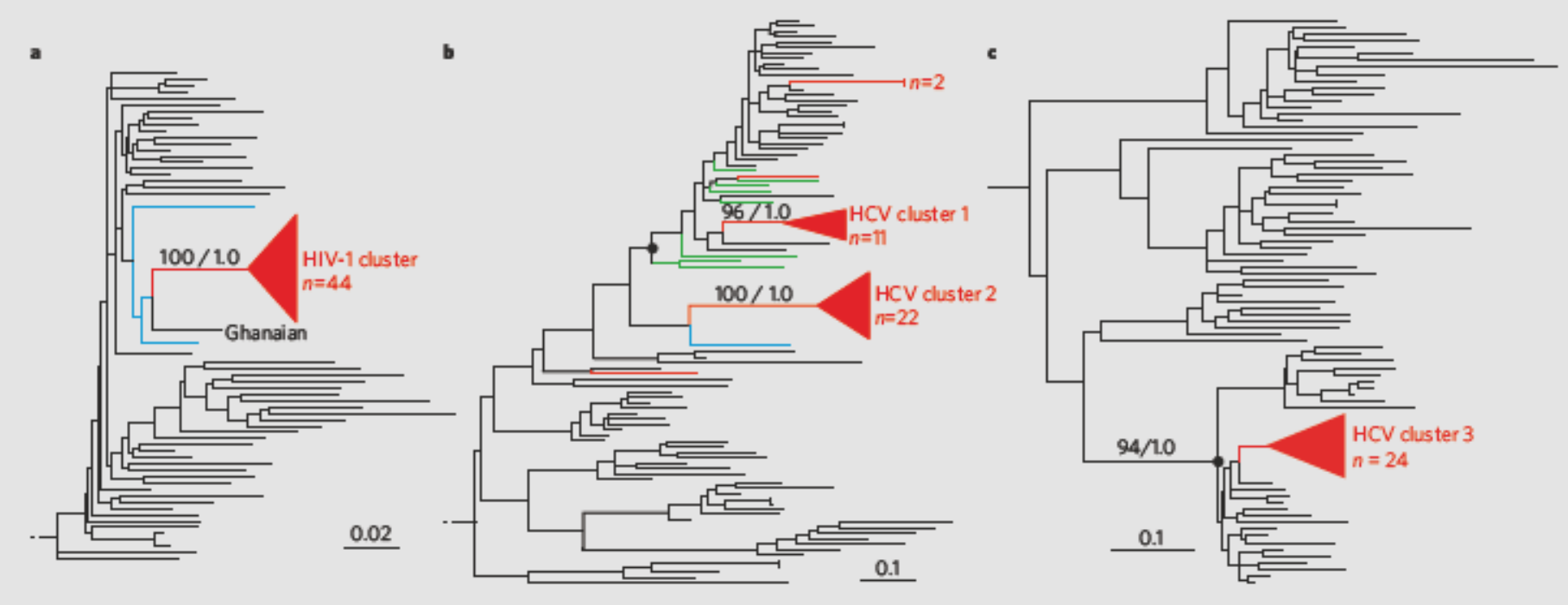

Figure 1| HIV-1 and HCV sequences from 1998 Al-Fateh Hos pit al (AFH) outbreak. a-c, Estimated maximum-likelihood phylogenies for HIV-1 CRF02_AG (a), HCV genotype 4 (b) and HCV genotype 1 (c). Source of sequences used for analysis: AFH, red; Egypt, green; Cameroon, blue. Black circles mark the common ancestor of HCV subtype 4a and 1a; numbers above AFH lineages give clade support values using bootstrap and bayesian methods, respectively. Scale bar units are nucleotide substitutions per site. For visual clarity, AFH clusters are represented by triangles and some noninformative reference strains are excluded. 
greater number of HCV clusters than HIV-1 clusters reflect the higher transmissibility of HCV compared with HIV-1 by such routes ${ }^{12}$. Crucially, we have shown that the HIV-1 and $\mathrm{HCV}$ strains responsible were being spread and transmitted among individuals attending the hospital before March 1998, indicating that many of the transmissions giving rise to the infection clusters must have already occurred before the foreign medical staff arrived. Tulio de Oliveira ${ }^{\star}$, Oliver G. Pybus ${ }^{*}$, Andrew Rambautt, Marco Salemił, Sharon Cassol\$, Massimo Ciccozzi||, Giovanni Rezza||, Guido Castelli Gattinaraף, Roberta D'Arrigo\#, Massimo Amicosante ${ }^{\star \star}$, Luc Perrint†, Vittorio Colizził Carlo Federico Pernoss, Benghazi Study Group IIII

*Department of Zoology, University of Oxford, Oxford OX13PS, UK

e-mait: tulio.deoliveira@zoo.ox.ac.uk †Institute of Evolutionary Biology, University of Edinburgh, Edinburgh EH93JT, UK †College of Medicine, University of Florida, Gainesville, Florida 32610, USA §Faculty of Health Sciences, University of Pretoria, 0001, Pretoria, South Africa ||Department of Infectious Diseases, Istituto Superiore di Sanità, 00161 Rome, Italy ๆDepartment of Immunology and Infectious Diseases, Bambino Gesù Children's Hospital, 00165 Rome, Italy \#Spallanzani Institute for Infectious Diseases, 00149 Rome, Italy

**Department of Internal Medicine, University of Rome "Tor Vergata", 00133 Rome, Italy ††Geneva University Hospital, 1211, Geneva, Switzerland

\#Department of Biology, University of Rome "Tor Vergata", 00133 Rame, Italy

§૬Department of ExperimentalMedicine and Biochemical Sciences, University of Rome "Tor

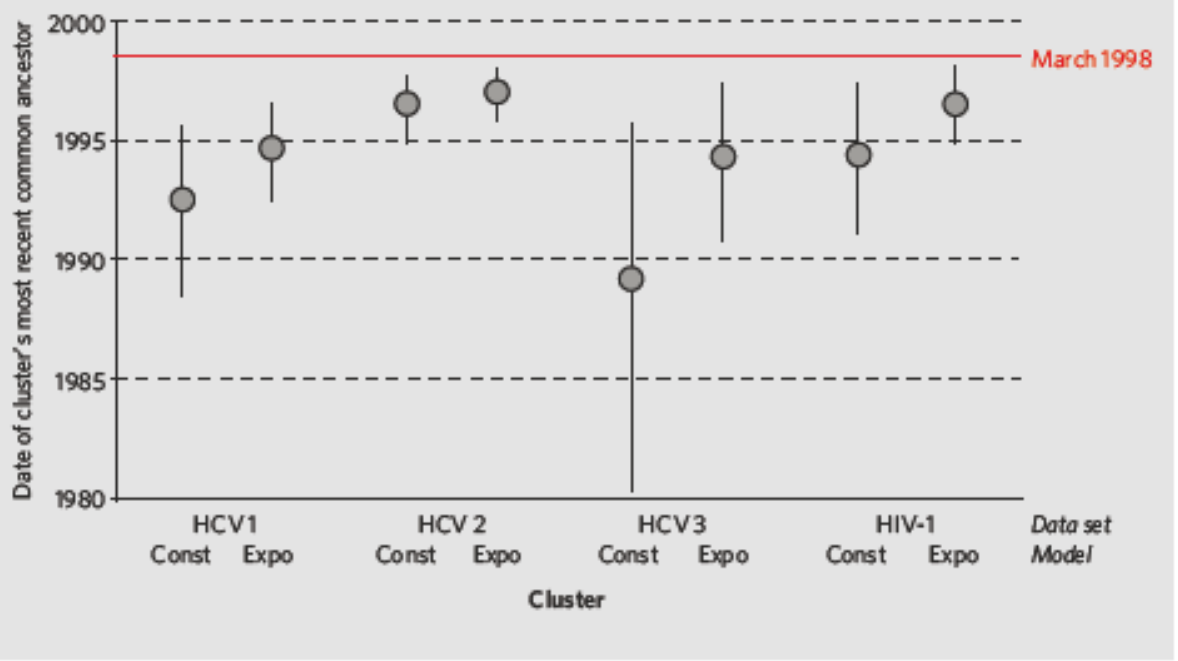

Figure 2 | Estimated dates of the most recent common ancestor for each cluster. Results obtained by using different evolutionary models. Vertical lines show the $95 \%$ highest posterior density intervals. Red line shows time of arrival of the foreign staff in March 1998. For further details, see supplementary information. 'Const', constant size; 'Expo', exponential growth.

Vergata", 00173 Rome, Italy

||||For full listing see supplementary information.

1. Yerly, S. etal. J.Infect. Dis.184, 369-372(2001).

2. Visco-Comandini, U. et al. AIDS Res. Hum. Retrovir. 18, $727-732$ (2002).

3. Human Rights Watch Stemming the Flow VoL 18, No. 5 (2006); http./fwwwhrw.org/reports/2006/libya0906

4. European Commission TechnicalMission to Libya on Mlegal Immigration, 27 Nov-6 Dec2004 (2006); http://www. statewatchorg/news/2005/may/eu-report-libya-illimmpdf

5. Ndjomou,J_Pybus, O.G.\&. Matz, B. J. Gen Vrol.84, 2333-2341 (2008).

6. Smith, D. B. etal.J. Gen. Viral. 78, 321-328 (1997).

7. Pybus, Q G et al. Sdence 292, 2323-2325 (2001)

8. Frank, C et al Lancet 355, 887-891(2000).

9. Pybus, Q G, Drummond A.L, Nakanq, T, Robertson, B.H.
\& Rambaut,A. Mol. Bial. Eval. 20, 381-387 (2008)

10. Drummond, A.J.Ho, S. Y, Phillips, M.J.\& Rambaut, A. PLOS Biol. 4, e88 (2006).

11. Montagnier, L.\& Colizzi, V. Report an the Benghazi Outbreak (2006); http://wwwnaturecom/hature/ journal/443/n7114/extref/montagnierpdf

12. Goldmann, D. A. L. Allergy Clin. Inmunal. 110 (suppL), 21-26(2002).

Supplementary information accompanies this communication on Nature's website. Received 4 November; accepted 24 November 2006. Published online 6December 2006. Competing financial interests: declared none doi: $10.1038 /$ nature $444836 a$

BRIEF COMMUNICATIONS ARISING online www.nature com/bca see Nature contents. 